\title{
Unusual clinical scenarios in Urology and Andrology
}

\author{
Lucio Dell'Atti ${ }^{1}$, Andrea Fabiani ${ }^{2}$, Erika Palagonia ${ }^{1}$, Agostini Edoardo ${ }^{1}$, Maria Pia Pavia ${ }^{2}$, \\ Simone Scarcella ${ }^{1}$, Valentina Maurelli ${ }^{2}$, Emanuele Principi ${ }^{2}$, Marco Tiroli ${ }^{1}$, Giulio Milanese ${ }^{1}$, \\ Lucilla Servi $^{2}$, Andrea Benedetto Galosi ${ }^{1}$ \\ ${ }^{1}$ Division of Urology, University Hospital "Ospedali Riuniti”, School of Medicine, Department of Clinical, Special and Dental \\ Sciences, Marche Polytechnic University, Ancona, Italy; \\ ${ }^{2}$ Urology Unit, Surgery Department, Macerata Civic Hospital, Area Vasta 3 Asur Marche, Italy.
}

\section{Summary This collection includes some unusual cases and how they were diagnosed and treated.}

Case 1: A case of a patient with primary hyperthyroidism presenting with a submucosal ureteral stone after endoscopic lithotripsy was described. After multiple endoscopic treatment, the stone was successfully removed by open ureterolithotomy recovering ureteral patency and normal renal function.

Case 2: A case of burned-out testicular cancer with atypical lymphatic spread (stage II A) was presented. After right orchiectomy and complete remission of tumor markers, due to atypical metastases location and uncertain histology, the patient was treated with systemic therapy based on bleomycin, etoposide and cisplatin (PEB). At re-staging after chemotherapy computed tomography showed reduction of all node metastases and an observation protocol was proposed.

Case 3: A patient was readmitted to hospital after 12 days from an uneventful Robot-Assisted Radical Prostatectomy (RARP) for prostate cancer due to lower abdominal pain plus abdominal distension, nausea and constipation not responsive to medical therapy. Computed Tomography showed colon and small bowel dilatation without any evidence of anatomical or mechanical obstruction. Laparoscopic abdominal exploration confirmed bowel distension without evidence of obstructing lesions. Ogilvie's Syndrome or acute colonic pseudo-obstruction (ACPO) was diagnosed. The patient fully recovered and was discharged six days after the procedure.

Case 4: A case of recurrent Acute Idiopathic Scrotal Edema (AISE) was diagnosed on clinical signs together with the decisive help of pathognomonic ultrasound findings as the "fountain sign".

Case 5: Small bilateral testicular nodules were diagnosed in a 30-years old patient undergoing scrotal ultrasound in follow up of acute lymphoblastic leukemia. Ultrasound guided testis sparing surgery was performed demonstrating Leydig cell tumors.

KEY WORDS: Submucosal ureteral stone; Burened-out testicular cancer; Ogilvie's Syndrome; Acute Colonic Pseudo-Obstruction (ACPO); Acute Idiopathic Scrotal Edema (AISE); Ultrasound guided testis sparing surgery.

Submitted 9 January 2021; Accepted 21 January 2021
Case 1.

Complication management OF atypical SUBMUCOSAL URETERAL STONE LOCALIZATION AFTER MULTIPLE ENDOSCOPIC TREATMENTS IN A PATIENT WITH ELEVATED PARATHYROID HORMONE LEVELS (Erika Palagonia, Marco Tiroli, Lucio Dell'Atti, Andrea Benedetto Galosi)

\section{INTRODUCTION}

Submucosal ureteral stone is a very atypical location and rare condition that can occur after multiple endoscopic procedures. In the literature, there is a complete lack of evidence in the management of this condition.

The European Association of Urology guidelines describe an overall complication rate after retrograde ureterorenoscopy (URS) ranging between 9\% and 25\% (1). The most frequent complications reported in the literature are: fever and sepsis, steinstrasse and ureteral lesions (2).

\section{Case report}

A 24-years-old man was referred to our urology department due to recurrent right flank pain, not amenable with medical therapy, within two weeks from endoscopic lithotripsy with rigid URS for ureteral stone. Blood test analysis showed a serum creatinine level of $1.28 \mathrm{mg} / \mathrm{dl}$ with an estimated glomerular filtration rate (eGFR) of 73 $\mathrm{ml} / \mathrm{min} / 1.73 \mathrm{~m}^{2}$, white cells count of $13.39 \times 10^{3} / \mathrm{mmc}$, and remaining indexes within the range of normality. A non-contrast enhanced Computed Tomography scan of the abdomen described a grade IV hydroureteronephrosis due to a $1.9 \mathrm{~cm}$ lumbar ureteral stone with concomitant inflammation and edema of perirenal tissues. A subsequent Renal Scintigraphy with Technetium ( ${ }^{99 \mathrm{~m} T C)}$ dimercaptosuccinic acid was performed defining a residual function of $31.6 \%$ for the right renal unit.

A right URS was attempted without success due to edema and extensive phlogistic reaction of ureteral orifice and a $26 \mathrm{~cm} 6 \mathrm{Ch}$ double J ureteral stent (DJS) was placed to drain the renal unit. The patient was discharged the next day and scheduled for a second URS treatment. Within 3 months the patient was readmitted due to abdominal right flank pain non-responsive to medical treatment despite the presence of DJS.

Fluoroscopy and retrograde pyelography showed both a proximal calcification of the right DJS and the ureteral 
stone located at lumbar level. In the light of the impossibility of an endoscopic removal of the JJ stent, the patient was submitted to an extracorporeal shock wave lithotripsy (ESWL) session to fragment the calcified proximal part of the DJS with 1600 shocks at maximum power. The DJS was then removed and a subsequent URS was performed to complete endoscopically the lithotripsy of residual calcified fragments and to treat the ureteral stone. Under direct endoscopic view of the ureter no intraluminal stone was evidenced. However, the radiogram of the abdomen evidenced the presence of a ureteral stone located at the level of the right lumbar ureter.

A multidisciplinary meeting was held with nephrologist to decide the appropriate treatment and due to the worsening of renal function and overall clinical conditions an open laparotomy surgery was scheduled. Intraoperatively an uretero-lithotomy was necessary to expose the submucosal ureteral stone. A longitudinal incision of the ureter allowed the extraction of the stone and a replacement of the $26 \mathrm{~cm}$ X 6 Ch DJS. The chemical and physical analysis of the stone showed a medium hard stone composed of calcium oxalate and calcium magnesium phosphate.

General condition of the patient rapidly improved with both resolution of symptoms and improvement of blood test analysis: serum creatinine level of $1 \mathrm{mg} / \mathrm{dl}$ and eGFR of $98 \mathrm{ml} / \mathrm{min} / 1,73 \mathrm{~m}^{2}$. Further laboratory investigations showed a serum calcium level of $11.2 \mathrm{mg} / \mathrm{dl}$ and a parathyroid hormone (PTH) level of $196 \mathrm{pg} / \mathrm{ml}$ (14-85 $\mathrm{pg} / \mathrm{ml}$ ). Due to the elevated levels of the PTH level an endocrinologist evaluation was requested. The right DJS was removed within 20 days from the procedure. Retrograde pyelography did not show ureteral strictures or ureteral stone recurrences and absence of hydronephrosis. After 1 year of follow up abdomen ultrasound showed absence of hydronephrosis and the presence of a small $5 \mathrm{~mm}$ stone in the lower pole of the right kidney. Endocrinological investigation demonstrated a primary hyperparathyroidism; the parathyroid scintigraphy with Technetium ${ }^{99 \mathrm{~m}} \mathrm{TC}$ ) sestamibi and ${ }^{99 m} \mathrm{TC}$ pertechnetate showed a hyper-capturing area in the lower lobe of the right portion of the thyroid lodge, suggestive of a hyperfunctioning parathyroid gland.

The patient was scheduled for surgical treatment.

\section{Conclusions}

Systematic review of literature on this argument showed limited evidence. Major complications following endoscopic surgery are potentially numerous and extremely varied, but submucosal ureteral stone localization was not found in any of the studies considered.

In our case we believed that the atypical localization of the stone is related to the specific clinical condition of the patient. A condition of primary hyperparathyroidism can lead to an increased risk of calcification in the kidney and ureter, especially in case of concomitant other factors such as infections, changes in urinary $\mathrm{pH}$ and the presence of proteins and electrolytes (3). Identifying patients at risk of complications after endoscopic treatments is essential to reduce the need of re-intervention. A widely accepted protocol including management of rare complications could represent a topic of great interest for the urological community to guide the best practice.

\section{REFERENCES}

1. Türk C, Petrík A, Sarica K, Seitz C, et al. EAU Guidelines on Interventional Treatment for Urolithiasis. Eur Urol. 2016; 69:475482.

2. Cindolo L, Castellan P, Primiceri G, et al. Life-Threatening complications after ureteroscopy for urinary stones: Survey and systematic literature review. Minerva Urol Nefrol. 2017; 69:421-431.

3. Dell'Atti L, Papa S. Ten-year experience in the management of distal ureteral stones greater than $10 \mathrm{~mm}$ in size. G Chir. 2016; 37:27-30.

Case 2.

Clinical stage IIA burned-out testicular CANCER WITH ATYPICAL LYMPHATIC SPREAD: A THREATENING CLINICAL SCENARIO (Edoardo Agostini, Giulio Milanese, Lucio Dell'Atti, Andrea Benedetto Galosi)

\section{INTRODUCTION}

Burned out testicular neoplasm is a rare form of cancer accounting $5 \%$ of all germ cell tumors (GCT). GCT clinical stage (CS) IIA is defined as any T stage with lymph node metastases less than $2 \mathrm{~cm}$ (cN1), with or without stage I tumor markers.

Depending on main histology and markers level, international guidelines suggest different treatments, ranging from radiotherapy to chemotherapy and retroperitoneal lymph-node dissection (RPLND). Optimal management in these patients is still a matter of debate. Furthermore, a critical feature in treatment choice is the metastatic pattern to the retroperitoneum lymph-nodes. We report the threatening case of a CS IIA burned out right testicular cancer with negativized markers and atypical metastatic spread.

\section{Case report}

We report the case of a 44 years-old man referred at our institution for a localized right scrotal pain. Ultrasound using a $10 \mathrm{MHz}$ linear probe was performed and revealed a $3 \mathrm{~cm}$ well-defined highly echogenic lesion with calcified areas in the right testis. Preoperative tumor markers were slightly raised: AFP $17 \mathrm{ng} / \mathrm{mL}$, HCG $52 \mathrm{mlU} / \mathrm{mL}$, LDH 165.

The patient underwent right orchiectomy, and histological specimens revealed regressed GCT. Chest and abdomen computed tomography (CT) scan performed after surgery showed retroperitoneal pathologically enlarged lymph nodes on the left side of aorta, a little cranially to common iliac arteries origin. The patient repeated tumor markers 10,15 and 25 days after surgery, showing complete remission since the first analysis. Due to atypical metastases location and uncertain histology, we advised for treating patient with systemic therapy based on bleomycin, etoposide and cisplatin (PEB). Three months after systemic therapy, we re-staged the patient with contrast enhanced chest-abdomen CT scan, showing reduction of all node metastases, now measuring less than $1 \mathrm{~cm}$ each. As the largest part of small residual masses after systemic therapy usually contain fibronecrotic tissue, we proposed the patient for observation protocol. 


\section{Discussion}

Burned out tumor, also named regressed tumor, is a germ cell tumor that has completely or partially regressed, leaving a scar in the testicular parenchyma with vestiges of GCT. It accounts 5\% of all GCT. Pure seminoma is considered the main histology presenting with regressed aspect.

The mechanism behind tumor regression is mainly attributed to immunological response by cytotoxic $\mathrm{T}$ lymphocytes or ischemic injury. Diagnosis is often incidental (ultrasound) or secondary to symptoms of metastatic spread. About primary tumor, a general disorganization of the sound pattern of parenchyma and focal/diffuse hypoechoic lesions are common features, with or without calcifications (1).

In 2017 was described a new pattern consisting of welldefined highly echogenic lesion with calcified areas resembling a pearl-oyster (2). In our report, staging by imaging revealed a clinical stage IIA with negative markers and with exclusively contralateral metastatic pattern. Past elegant studies about retroperitoneum lymphatic supply, showed that right testis drains primarily to the interaortocaval nodes with some drainage to the right paracaval nodes.

Only in some studies a small but appreciable amount of lymphatic drainage from the right testis was found draining to left para-aortic region. About treatment, clinical stage IIA, particularly marker-negative non-seminoma, is actually one of main concern in testicular cancer management. However, National Comprehensive Cancer Network (NCCN), American Urological Association (AUA) and European Association of Urology (EAU) guidelines agree in recommending chemotherapy as possible firstline treatment for patients with marker-negative clinical stage IIA non seminoma germ cell tumor (NSGCT) of the testicle (3).

Criteria as larger or multiple lymph nodes or metastatic lesions outside the primary landing zone (as in our patient) can be useful in treatment choice.

\section{Conclusions}

Treatment of CS IIA GCT with negative markers represents a recurrent impasse for the clinician, with different possible first line effective treatments, depending on primary histopathology and markers. In our case we present this intricate situation, complicated by atypical lymphatic spread and rare histologic specimen (with typical ultrasound pattern). In this threatening setting, we could establish the effectiveness of chemotherapy in this scenario and the usefulness of criteria as metastatic spread pattern for the choice of treatment.

\section{References}

1. Angulo JC, González J, Rodríguez N, et al. Clinicopathological study of regressed testicular tumors (apparent extragonadal germ cell neoplasms). J Urol. 2009; 182:2303-2310.

2. Dell'Atti L, Galosi AB. "Pearl oyster": a new ultrasonographic sign of the regressed testicular tumor. J BUON. 2017; 22:1610-1611

3. Honecker F, Aparicio J, Berney D, et al. ESMO Consensus Conference on testicular germ cell cancer: diagnosis, treatment and follow-up. Ann Oncol. 2018; 29:1658-1686.

\section{Case 3.}

OGILVIE'S SYNDROME IN POST ROBOT-ASSISTED RADICAL PROSTATECTOMY: THE IMPORTANCE OF DIFFERENTIAL DIAGNOSIS AND SURGICAL TREATMENT (Simone Scarcella, Lucio Dell'Atti, Giulio Milanese, Andrea Benedetto Galosi)

\section{INTRODUCTION}

Ogilvie's Syndrome or acute colonic pseudo-obstruction (ACPO) is characterized by massive non-toxic acute intestinal distension in the absence of clear anatomical or mechanical obstructions. It was first described by Sir William Ogilvie in 1948 (1). Throughout academic literature, numerous articles regarding ACPO have been published describing this rare occurrence after general abdominal surgery, spinal surgery and multiple trauma surgeries in patients with an average onset age of 64-74 years (2). However, reviewing all published articles, only few cases have been reported after genitourinary surgeries (3). Certain risk factors have widely been accepted such as a recent major surgery, even in the absence of abdominal involvement, pregnancy, pre-existing medical comorbidities and coexistence of multiple precipitating conditions (systemic illnesses, infections, diabetes or cardiovascular diseases) $(4,5)$. Despite this recognition, there is a substantial lack of a specific etiology and this phenomenon is classified as a disorder of the intestinal motility due to dysfunction of the interstitial cells of the enteric nervous system and concurrent autonomic imbalance. Bowel injuries rarely occur in robot-assisted radical prostatectomy during intestinal manipulation or instruments introduction. Ogilvie's Syndrome is a sporadic, under-reported diagnosis of exclusion. It can mimic symptoms of intestinal ischemia or perforation but the appropriate treatments of these conditions are different. In this setting, an accurate differential diagnosis is mandatory and atypical cases of colonic obstruction should always raise suspicion. We encountered a case of a patient who developed Ogilvie's Syndrome after an uneventful robot-assisted radical prostatectomy (RARP), requiring laparoscopic abdominal explorative surgery.

\section{Case report}

A 65 years-old patient with no previous medical history was scheduled at our urological unit for Robot-Assisted Radical Prostatectomy (RARP) to treat an organ confined 3+4 = 7 Gleason Score (Grade Group 2) prostate cancer. PSA was $12 \mathrm{ng} / \mathrm{ml}$ and further radiological evaluation through abdominal computed tomography and bones scintigraphy resulted negative for both lymphatic spread and bone metastasis. Briganti's nomogram score for suspected lymphatic invasion resulted $3.5 \%$ with no indication to perform lymphadenectomy. A six-port approach with open Hasson optical trocar placement was used with instruments insertion completed under direct vision to avoid bowel injuries, as routinely performed by our team during robot assisted and pure laparoscopic surgeries. No intestinal lesion was suspected during the surgery and the procedure was completed uneventfully, with bilateral nerve sparing technique and no lymphadenectomy, by a senior urologist with over twenty years' experience. Within three days the patient was dis- 
charged with regular intestinal canalization and blood test panel in normal range. After 12 days from RARP he was re-admitted to our institution for lower abdominal pain plus abdominal distension, nausea and constipation not responsive to medical therapy. A full blood test panel showed leukocytosis and elevated acute phase reactants with normal renal function, urea and electrolytes ruling out an electrolyte imbalance and the possibility of a chemical ileus as potential cause for the clinical findings. A consultation of a general surgeon was requested and prompt conservative management was started. Presuming a bowel sub-obstruction, the attending general surgeon prescribed bowel rest, nasogastric and rectal drainage associated with the infusion of metoclopramide to treat nausea and for its prokinetic intestinal effect. Intravenous empiric antibiotic therapy with co-amoxiclavulanate and metronidazole was started to prevent potential intra-abdominal sepsis. After an initial improvement of symptoms both blood test analysis and clinical condition of the patient worsen and an abdominal Computed Tomography scan (CT) was planned. It showed a dilated caecum with collapsing descending colon and rectum without any evidence of anatomical or mechanical obstruction. Despite the presence of linear pneumatosis within the ascending colon to the hepatic flexure, raising suspect of intestinal ischemia and perforation, no clear evidence of obstructive occlusion was observed (Figure 1). The patient clinically deteriorated and accordingly with the radiological findings of suspected visceral rupture and clinical symptoms of peritonitis an emergency laparoscopic explorative abdominal surgery was performed. It confirmed small bowel and colonic distension with a small amount of free fluid in the pelvis, but all intestinal structures resulted viable without evidence of obstructing lesions. Only minor adherences were detected and incised within the small

Figure 1 - Case 3.

CT of the abdomen showing colonic and small bowel dilatation.
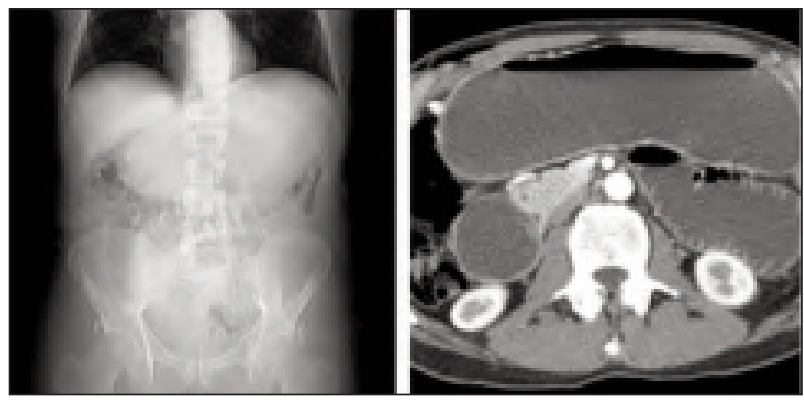

Table 1 - Case 3.

Summary of differential diagnosis between acute colonic pseudo-obstruction (ACPO) and bowel obstruction.

\begin{tabular}{|ll|}
\hline Mechanical - dynamic & Functional - adynamic \\
\hline -Mural (malignant/inflammatory stricture) & - Ileus \\
\hline -Extramural (congenital bands/adhesions, volvulus) & - Acute mesenteric arterial occlusion \\
\hline- Intraluminal (colorectal carcinoma) & - Mesenteric vein thrombosis \\
\hline -Infective colitides & \\
\hline -Inflammatory bowel disease & \\
\hline
\end{tabular}

bowel. Both stool cultures for infective colitis and additional virology blood screening resulted negative. Within three days the abdominal drain was removed and the patient was discharged six days from the procedure, after complete recovery.

From the combination of radiological, microbiological and clinical data an APCO diagnosis was determined, having excluded other causes of both functional and mechanical bowel obstruction (Table 1).

\section{Conclusions}

With this case report we aim to raise awareness of Ogilvie's Syndrome. It remains a sporadic and underreported occurrence of intestinal motility impairment with no defined causes within the urological community. It mimics obstructive symptoms without any definable mechanical obstruction, leading to massive colonic and small bowel intestinal distension. Supportive medical management aims to prevent progression to peritonitis and intestinal perforation. In patients failing to respond to conservative approaches urgent surgical intervention is mandatory, to minimize morbidity and mortality.

\section{References}

1. Ogilvie H. Large-intestine colic due to sympathetic deprivation; a new clinical syndrome. Br Med J. 1948; 2:671-673.

2. Wegener M, Borsh G. Acute colonic pseudo-obstruction (Ogilvie's Syndrome). Presentation of 14 of our own cases and analysis of 1027 cases reported in the literature. Surg Endosc. 1987; 1:169-174.

3. Kevin T.McVary, Daniel P. Dalton and Michael D.Blum. Acute Intestinal Pseudo-Obstruction (Ogilvie's Syndrome) complicating Radical Retropubic Prostatectomy. J Urol. 1989; 141:1210-1212.

4. Vanek VW, Al-Salti M. Acute pseudo-obstruction of the colon (Ogilvie's Syndrome). An analysis of 400 cases. Dis Colon Rectum. 1986; 29:203-210.

5. Galosi AB, Dell'Atti L, Bertaccini A, et al. Clinical evaluation of the iXip index to reduce prostate re-biopsies. Cancer Treat Res Commun. 2018; 16:59-63.

\section{Case 4.}

SONOGRAPHIC FINDING OF "FOUNTAIN SIGN" IN ERYTHEMATOUS SCROTUM: A CASE OF RECURRENT ACUTE IDIOPATHIC SCROTAL EDEMA

(Maria Pia Pavia, Andrea Fabiani, Emanuele Principi, Lucilla Servi)

\section{INTRODUCTION}

Acute Idiopathic Scrotal Edema (AISE) is a self-limiting cause of acute scrotum that mainly affects child population. We present a case of recurrent AISE, which diagnosis was made based on clinical signs together with the decisive help of pathognomonic ultrasound findings as the "fountain sign".

\section{Case report}

A nine-year-old patient presented to the Emergency Department with a 6-hour hystory of bilateral scrotal dyscomfort, swelling and redness. Blood test and urinalysis were normal. He was referred to our Urology Unit. 
The patient was afebrile and asymptomatic. Previous medical history was unremarkable. On clinical examination, the scrotum was enlarged but painless and the skin was red and tender at palpation (Figure 1). The child underwent scrotal ultrasound (US) scan demonstrating the absence of hydrocele and showing an hyperemic thickened scrotal wall around the testicles in trasverse scan (Figure 2).

Color Doppler US showed normal appearance of both testis and epydydimis. Diagnosis of AISE was made. The patient was discharged with anti-inflammatory therapy improving in a few days. One month later, he returned to the Emergency Department presenting the same symptoms. Clinical and ultrasonographic findings were stackable. The mother reported that he woked up on morning with swollen lips. Diagnosis of AISE was confirmed. The patient was referred to pediatrics for internal evaluation work-up.

AISE is defined as a self-limiting condition that mainly affects children between ages 5 to 11 and accounts for $>10 \%$ of cases of acute scrotum in childhood. Diagnosis of AISE made in the original reports was reviewed by Santi et al. according to the following criteria: acute onset of redness and swelling of the scrotal sac produced by subcutanous edema, associated with normal testes, after exclusion of further possible cause of acute scrotum (1). The etiology is still unknown and recurrences are reported in approximately $10 \%$ of cases. It has been suggested that AISE is common among patients with atopic diathesis and it could represent a hypersensitivity reaction related to a angioneurotic edema's variant. In our case,

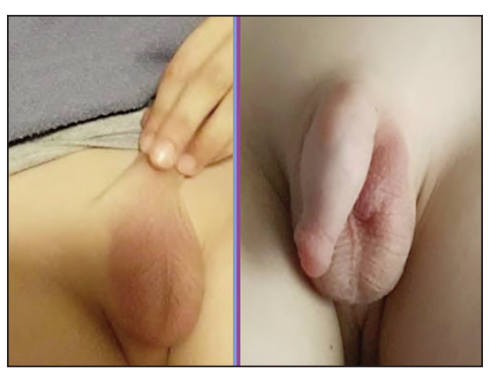

Figure 1 - Case 4.

First (left) and recurrent (right) presentation of AISE: Erythema and redness on the anterior surface of the scrotum.

Figure 2 - Case 4.

The patognomonic "fountain sign": Hypervascularity of the interscrotal septum was configured as an increased Color Doppler Signal resembling a fountatin at the transverse scan.

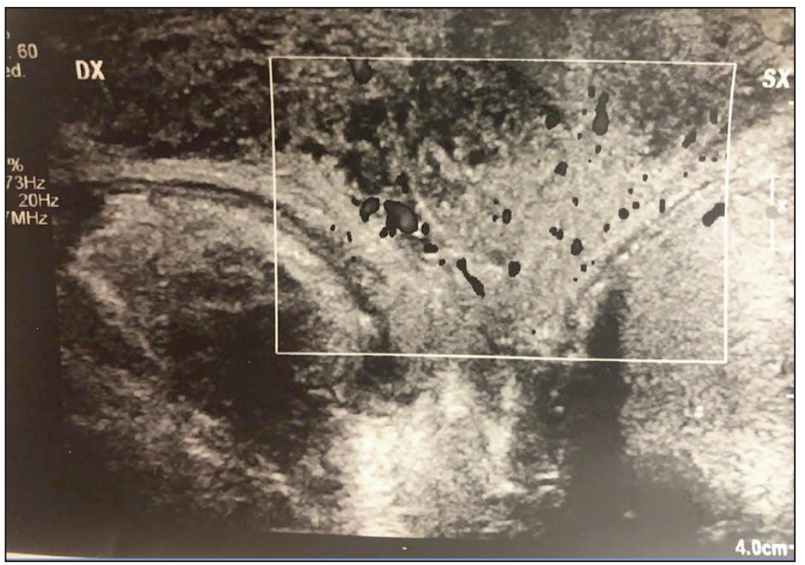

the patient experienced swelling of the lips which could confirm the correlation with this disease. However, laboratory tests were normal at all. Diagnosis is defined as clinically based, but as in our case it may be very difficult to make a certain diagnosis based on clinical findings.

Reaching an accurate and timely diagnosis is paramount, as it allows to exclude testicular malignancies or diseases requiring urgent care. Ultrasonography is the first-line imaging modality to evaluate the acute scrotum.

Combined with anamnesis and clinical examination, sonographic results become even more helpful when testicular torsion is highly suspected (2). To date, very few papers underlined the role of ultrasonography in the management of the disease.

Lee et al. stressed the importance of ultrasonography in managing this disorder, describing characteristic US and Doppler imaging findings that may help to avoid unnecessary surgical indication $(3,4)$.

The homogeneous thickening and edema of the scrotal wall together with hyperemia of the scrotum were described in previous studies as specific sonographic findings for AISE. The "fountain sign" was first described by Geiger et al. (5). Similarly, in our case we noticed a marked thickening of the scrotal skin characterized by an inhomogeneous US echostructure and an increase in the vascular texture to the Color Doppler Scan (CDS); at the transverse CDS, the intense blood supply to the interscrotal septum was configured as an increased Color Doppler signal that resembles a fountain. Sonographic finding of "fountain sign" was the only one that allowed us to make a certain diagnosis of AISE. In their report, Patoulias et al. also highlighted the role of US in the AISE diagnosis, stigmatizing the ongoing uncertainty of the diagnosis of exclusion and stressing the need to diagnose the disease based on pathognomic ultrasound signs.

\section{Conclusions}

AISE is not such a rare condition; the fast and self-limiting resolution underestimates its incidence and limits clarity about its possible etiology. For this reason, the constant use of ultrasonography allows a more rapid and certain clinical diagnosis. However, it has to be proven if the systematic search for the pathognomonic sign can help to define the real spread of the condition and ensure more information about its etiology. It is no longer relevant to define AISE as a diagnosis of exclusion. Diagnosis can be certainly made through the sonographic finding of "fountain sign", which is the diagnostic key of the disease.

\section{REFERENCES}

1. Santi M, Lava SAG, Simonetti GD, et al. Acute Idiopathic Scrotal Edema: Systematic Literature Review. Eur J Pediatr Surg. 2018; 28:222-226.

2. Martino P, Galosi AB, Bitelli M, et al. Imaging Working GroupSocieta Italiana Urologia (SIU); Società Italiana Ecografia Urologica Andrologica Nefrologica (SIEUN). Practical recommendations for performing ultrasound scanning in the urological and andrological fields. Arch Ital Urol Androl. 2014; 86:56-78.

3. Lee A, Park SJ, Lee HK, et al. Acute idiopathic scrotal edema: ultrasonographic findings at an emergency unit. Eur Radiol. 2009; 19:2075-2080 
4. Dell'Atti L. Successful management of an asymptomatic bilateral synchronous testicular carcinoid tumor with a testicular-sparing surgery. Asian J Androl. 2017; 19:507-508.

5. Patoulias D, Rafailidis V, Feidantsis T, et al. Fountain's sign as a diagnostic key in acute idiopathic scrotal edema: case report and review of the literature. Acta Medica (Hradec Kralove). 2018; 61:37-39.

\section{Case 5.}

ULTRASOUND INTRAOPERATIVE MANAGEMENT OF BILATERAL LEYDIG CELL TUMOR IN A YOUNG PATIENT (Andrea Fabiani, Maria Pia Pavia, Valentina Maurelli)

\section{INTRODUCTION}

Testis ultrasound increased detection of small and not palpable testicular lesions. In small nodular lesions, ultrasound guided excision is mandatory. We report a case of a 30-years old patient undergoing an ultrasound guided testis sparing surgery for a small bilateral testicular lesion.

\section{Case report}

In April 2015, a young patient underwent a scrotal ultrasound evaluation in follow up of acute lymphoblastic leukemia treated by bone marrow transplantation in 2004. Two hypo-echoic small testicular nodules sized 5 $\mathrm{mm}$ and $3 \mathrm{~mm}$ were diagnosed in left testis mediastinum. In October 2015, at our Urologic Department, also a diagnosis of a right $5 \mathrm{~mm}$ nodular testicular lesion was made. Half yearly ultrasound follow up was performed until the evidence of progressive growth of one left nodules (diameter of $1.17 \mathrm{~cm}$ from $5 \mathrm{~mm}$ ) in 2020 . Male fertility diagnostic work up revealed a non-obstructive azoospermia. The oncologic markers were negative. We decided to perform a bilateral ultrasound guided excisional nodular testicular biopsy (UGENT) with an inguinal approach. After scrotum incision, ultrasound was used to identify the lesions. The nodules were marked with a 23-gauge needle (Figure 1). After incision of tunica albuginea, the testicular nodule was bluntly dissected and the surgical specimen was sent to frozen section examination. Diagnosis of Leydig cell tumor for each of three nodules was made. The tunica albuginea was closed. After tunica vaginalis eversion, both testes were replaced in the scrotum (Figure 2). Testis ultrasound evaluation was performed immediately after nodules excision. Ultrasound study of the testis, performed at post-operative day 1 , showed the complete disappearance of the lesions without evidence of intra-testicular

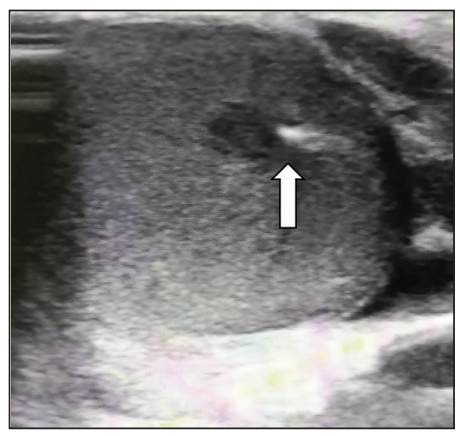

Figure 2 - Case 5.

Surgical procedure.

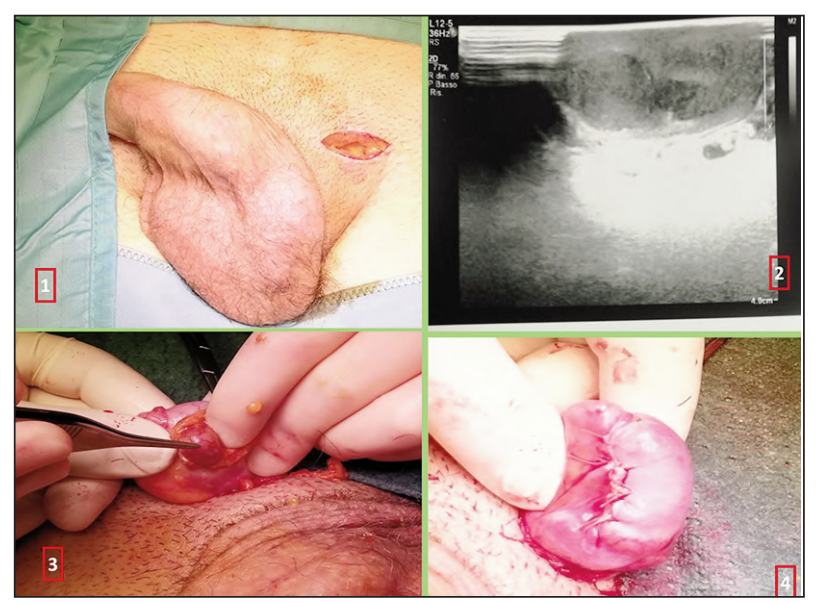

hematoma. Histopathologic exam diagnosed a Leydig cell tumor with surgical margins free from disease.

\section{Conclusions}

The detection of small and not palpable testicular lesions is increased due to the widespread use of scrotal ultrasound (1). Small testicular masses are often benign and do not always require radical orchidectomy. Preoperative ultrasound can assess lesion size and the smaller the nodule, the less likely that it is malignant (2). In fact, in selected patients, active ultrasound surveillance may obviate the need for surgical resection. Sub centimetric testicular lesions are more likely to be impalpable and are therefore usually detected incidentally on scrotal ultrasound performed for various kind of indications. For hypo-echoic lesions, as in our case, the size is an independent risk factor for malignancy and the risk of the malignancy increase with the lesion size (cut off value: $5 \mathrm{~mm}$ ) (2). However, nodular size is not always associated with the benign feature. As presented in our previous experience, in a series of 8 small testicular nodules $(<1 \mathrm{~cm}$ ), we found $37.5 \%$ of malignant tumors (4 $\mathrm{mm}, 6 \mathrm{~mm}, 8 \mathrm{~mm}$ ) (3). Ultrasound surveillance permits monitoring of lesion size, which may allow more accurate risk stratification. There is no consensus on the appropriate ultrasound surveillance protocol to follow up the small testicular lesions. Germ cell tumor doubling time has been reported to be 10 to 30 days based on which some authors recommend a surveillance interval of 3 months, but others applied a 6-month follow up strategy (2). It is also mandatory to underline the importance of intra-operative ultrasound in localization of the lesion, in order to permit the frozen section examination (FSE) of the complete excided entire nodule (3). Technical aspects of UGENT have been extensively illustrated in scientific literature with or without microsurgical approach, independently from use of advanced ultrasound techniques (2). As demonstrated in this case, the simple and low cost needle use (23 Gauge) allowed to locate the small nodular lesion and permitted the complete removal. The UGENT may be very difficult in case of very small nodules, especially in cases of lesions $<5$ $\mathrm{mm}$ (3), posing problems both in terms of sending a suf- 
ficient tissue amount to the FSE and for the absence of a sure resection margin. In all cases, also the close collaboration with the pathologist is very useful in reducing diagnostic and therapeutic errors (3).

Testicular sparing surgery is mandatory in case of benign lesions and also in monorchid.

This case highlights the importance of ultrasound testis follow up of small nodules, especially in young patient with infertility risk factors and, mostly, the role of intraoperative ultrasound in testicular sparing approach for small testicular lesions.

\section{REFERENCES}

1. Galosi AB, Fulvi P, Fabiani A, et al. Testicular sparing surgery in small testis masses: a multinstitutional experience. Arch Ital Urol Androl 2016; 4:320-324.

2. Dell'Atti L. Efficacy of ultrasound-guided testicle-sparing surgery for small testicular masses. J Ultrasound. 2015; 19:29-33.

3. Dell'Atti L, Fulvi P, Galosi AB. Are ultrasonographic measurements a reliable parameter to choose non-palpable testicular masses amenable to treatment with sparing surgery? J BUON. 2018; $23: 439-443$

\section{Correspondence}

Lucio Dell'Atti, MD, PhD (Corresponding Author)

dellatti@hotmail.com

Erika Palagonia

Marco Tiroli

Andrea Benedetto Galosi

Division of Urology, University Hospital "Ospedali Riuniti”, Marche Polytechnic University,

Via Conca 71, 60126 Ancona (Italy)

Lucio Dell'Atti, MD, PhD (Corresponding Author)

dellatti@hotmail.com

Agostini Edoardo

Giulio Milanese

Andrea Benedetto Galosi

Division of Urology, University Hospital "Ospedali Riuniti", Marche Polytechnic University,

Via Conca 71, 60126 Ancona (Italy)

Lucio Dell'Atti, MD, PhD (Corresponding Author)

dellatti@hotmail.com

Simone Scarcella

Giulio Milanese

Andrea Benedetto Galosi

Division of Urology, University Hospital "Ospedali Riuniti”, Marche Polytechnic University,

Via Conca 71, 60126 Ancona (Italy)

Maria Pia Pavia

Andrea Fabiani

andreadoc1@libero.it

Emanuele Principi

Lucilla Servi

Urology Unit, Surgery Department, Macerata Civic Hospital, Area Vasta 3 Asur Marche (Italy)

Andrea Fabiani

andreadocl@libero.it

Maria Pia Pavia

Valentina Maurelli

Urology Unit, Surgery Department, Macerata Civic Hospital, Area Vasta 3 Asur Marche (Italy) 